Int. J. Curr. Res. Med. Sci. (2017). 3(10): 98-106

\begin{tabular}{|c|c|c|}
\hline & International Journal of Current Research in & IICRMS \\
Medical Sciences & ISSN: 2454-5716 \\
\hline & P-ISJN: A4372-3064, E-ISJN:A4372-3061 \\
WWW.ijcrims.com & \\
\hline
\end{tabular}

\title{
Comparative study of sequential therapy versus standard triple therapy versus quinolone-based triple therapy for eradication of Helicobacter pylori infection
}

\author{
*Dr. Krishan kumar Oberoi, **Dr. Upasana \\ * Associate Professor, Department of Medicine, Govt. Medical College, Amritsar \\ **Lecturer, Department of Obstetrics and Gynaecology, Govt. Medical College, Amritsar \\ Corresponding Author: Dr. Krishan kumar Oberoi, Associate Professor, Department of Medicine, \\ Govt. Medical College, Amritsar. \\ E- mail:drkrishanoberoi@gmail.com
}

\begin{abstract}
Introduction: $H$. pylori is a gram-negative bacillus that has naturally colonized humans. Essentially all $H$. pyloricolonized persons have gastric tissue responses, but fewer than $15 \%$ develop associated illnesses such as peptic ulceration, gastric adenocarcinoma, or gastric lymphoma. Worldwide, more than $80 \%$ of duodenal ulcers and more than $60 \%$ of gastric ulcers are related to H. pylori colonization. Although $H$. pylori is susceptible to a wide range of antibiotics in vitro, monotherapy is not usually successful. Failure of monotherapy has prompted the development of multidrug regimens, the most successful of which are triple and quadruple combinations. Aims and Objectives: To compare efficacy of Sequential Therapy versus Standard Triple Therapy versus Quinolone-based Triple Therapy for eradication of Helicobacter Pylori infection. Methods: The study included 150 patients attending OPD / admitted in various wards of a tertiary care hospital diagnosed to be helicobacter pylori positive by rapid urease test, after obtaining informed consent. The patients were then be randomly divided into three groups each of 50, one had received Sequential Therapy, other triple therapy and third quinolone based therapy. Results and conclusion: Sequential therapy group had better eradication rates (90\%) as compared to standard triple therapy group (86\%) and fluroquinolone therapy group $(82 \%)$ but results were not statistically significant when all three groups were compared together.
\end{abstract}

Keywords: H. pylori, Rapid Urease test, Gastric lymphoma.

\section{Introduction}

Helicobacter pylori colonizes the stomachs of $50 \%$ of the world's human population throughout their lifetimes ${ }^{1}$. Colonization with this organism is the main risk factor for peptic ulceration as well as for gastric adenocarcinoma and gastric MALT (mucosa-associated lymphoid tissue) lymphoma ${ }^{2}$. Treatment for $\mathrm{H}$. pylori has revolutionized the management of peptic ulcer disease, providing a permanent cure in most cases. Such treatment also represents first-line therapy for patients with lowgrade gastric MALT lymphoma ${ }^{3}$. 
H. pylori is a gram-negative bacillus that has naturally colonized humans for at least 50,000 years-and probably throughout human evolution. It lives in gastric mucus, with a small proportion of the bacteria adherent to the mucosa and possibly a very small number of the organisms entering cells or penetrating the mucosa; its distribution is never systemic. The organism has several acid-resistance mechanisms, is slow-growing, and requires complex growth media in vitro ${ }^{4}$.

The prevalence of $H$. pylori among adults is $30 \%$ in the United States and other developed countries as opposed to more than $80 \%$ in most developing countries $^{5}$. The low incidence among children in developed countries at present is due, at least in part, to decreased maternal colonization and increased use of antibiotics. Humans are the only important reservoir of $\mathrm{H}$. pylori. Children may acquire the organism from their parents (more often from the mother) or from other children ${ }^{6}$.

H. pylori colonization induces a tissue response in the stomach, chronic superficial gastritis, which includes infiltration of the mucosa by both mononuclear and polymorphonuclear cells ${ }^{7}$. The pattern of gastric inflammation is associated with disease risk: antral-predominant gastritis is most closely linked with duodenal ulceration, whereas pangastritis is linked with gastric ulceration and adenocarcinoma. This difference probably explains why patients with duodenal ulceration are not at high risk of developing gastric adenocarcinoma later in life, despite being colonized by $H$. pylori.

Essentially all $H$. pylori-colonized persons have gastric tissue responses, but fewer than $15 \%$ develop associated illnesses such as peptic ulceration, gastric adenocarcinoma, or gastric lymphoma. Worldwide, more than $80 \%$ of duodenal ulcers and more than $60 \%$ of gastric ulcers are related to $H$. pylori colonization ${ }^{8}$. The main lines of evidence for an ulcer-promoting role for $H$. pylori are that (1) the presence of the organism is a risk factor for the development of ulcers, (2) non-NSAID-induced ulcers rarely develop in the absence of $H$. pylori, (3) eradication of $H$. pylori markedly reduces rates of ulcer relapse, and (4) experimental $H$. pylori infection of gerbils causes gastric ulceration.

Although $H$. pylori is susceptible to a wide range of antibiotics in vitro, monotherapy is not usually successful, probably because of inadequate antibiotic delivery to the colonization niche. Failure of monotherapy has prompted the development of multidrug regimens, the most successful of which are triple ${ }^{9,10,11}$ and quadruple combinations. Initially these regimens produced $H$. pylori eradication rates of more than $90 \%$ in many trials; in recent years, however, resistance to key antibiotics has become more common, a trend leading to $H$. pylori eradication rates of only 75-80\% for the most commonly used regimens. Current regimens consist of a PPI or $\mathrm{H}_{2}$ blocker, bismuth citrate ${ }^{12.13}$ and two or three antimicrobial agents given for 7-14 days. Research on optimizing drug combinations to increase efficacy continues, and it is likely that guidelines will change as the field develops and as countries increasingly individualize treatment to suit local antibiotic resistance patterns and economic needs. Resistance to clarithromycin and, to a lesser extent, to metronidazole are of growing concern. Clarithromycin resistance is less prevalent but, if present, usually results in treatment failure ${ }^{14}$. Therefore, an increasing number of patients require second theraputic attempt to eradicate the infection after treatment with triple drug.

Concomitant therapy is better for clarithromycinresistant strains, and 14 days of concomitant therapy is superior to 14-day triple therapy, with cure rates of $\geq 90 \%{ }^{15}$.

In view of the observation that $15-25 \%$ of patients treated with first-line therapy may still remain infected with the organism, new approaches to treatment have been explored. One promising approach is sequential therapy ${ }^{16}$. This regimen consists of 5 days of amoxicillin and a PPI, followed by an additional 5 days of PPI plus tinidazole and clarithromycin..Basis for this regimen is that by reducing bacterial load in first 5 days efficacy of tinidazole and clarithromycin increases. Initial studies have demonstrated eradication rates of more than $90 \%$ with good patient tolerance. 
Gatta et al. report a systematic review that identified 13 trials evaluating 3271 patients. Their data suggest that sequential therapy achieves 12 percent better absolute eradication rate than standard PPI triple therapy ${ }^{17}$.

In one meta-analysis of 10 randomized trails sequential therapy showed $93.4 \%$ success rate as compared to 76.9 for triple therapy. Most of studies are conducted in Italy. Results are also promising from Thailand, Spain, Taiwan. However, studies from Panama, France have failed to show any benefit ${ }^{18 .}$

Other promising approach is use of Quinolone based therapy consisting of Omeprazole $(20 \mathrm{mg}$ bid), Amoxicillin (1gm bid), Levofloxacin (500mg bid) for 10 days. In 2007 and 2009 Gisbert and colleagues from Spain published two prospective uncontrolled studies with 64 and 75 patients, respectively, evaluating the combination of levofloxacin $2 \times 500 \mathrm{mg}$ and amoxicillin together with ranitidine bismuth citrate or a PPI for 10 days. Eradication rates in both studies were similar at $84 \%$ and $83 \%{ }^{19}$. Because antibiotic resistance varies geographically it is essential to evaluate sequential therapy in this region of India.

\section{Aims \& Objectives}

To compare efficacy of Sequential Therapy versus Standard Triple Therapy versus Quinolone-based Triple Therapy for eradication of Helicobacter pylori infection.

\section{Materials and Methods}

The study included 150 patients attending OPD / admitted in various wards of a tertiary care hospital diagnosed to be helicobacter pylori positive by rapid urease test, after obtaining informed consent. The patients were then be randomly divided into three groups each of 50 , one had received Sequential Therapy with Omeprazole (20 mg) plus Amoxicillin (1 g) twice/day for five days, followed by Omeprazole $(20 \mathrm{mg})$ with Tinidazole $(500 \mathrm{mg})$ twice/day and Clarithromycin (500 $\mathrm{mg})$ twice/day for five consecutive days. Standard triple therapy group had received Omeprazole (20 mg), Amoxicillin (1g) and Clarithromycin (500mg) twice/day for 14 days and third group had received QuinoloneBased Triple Therapy Omeprazole (20mg bid), Amoxicillin (1 gm bid), Levofloxacin (500mg bid) for 10 days. Patients were followed up no sooner than four weeks of completing therapy by rapid urease test to confirm eradication. In cases of duodenal or gastric ulcers compelling continued use of proton-pump inhibitors after completion of antibiotic therapy, patients were followed up four weeks after stopping proton-pump inhibitors.

\section{Inclusion criteria:}

- Individuals of age more than 18 years age.

- Randomized after positive rapid urease test

\section{Exclusion criteria:}

- Chronic use of PPIs or H2-receptor antagonists

- Use of antibiotics in the previous two weeks

- Concomitant anticoagulant or nonsteroidal anti-inflammatory drug use

- Zollinger-Ellison syndrome

- Known allergy to the prescribed antibiotics

- Pregnant or breastfeeding women

- Severe or unstable cardiovascular

- Clinically significant renal or hepatic disease or dysfunction

- Any other clinically significant medical condition that could increase risk of side effects.

- Patients with Barrett's esophagus and high-grade dysplasia

- Patients with severe psychiatric or neurological disorder

- Eradication rates in two groups will then be analyzed statistically. 
Int. J. Curr. Res. Med. Sci. (2017). 3(10): 98-106

\section{Observations}

Table 1 Comparison of endoscopic diagnosis in three groups

\begin{tabular}{|l|c|c|c|c|}
\hline $\begin{array}{c}\text { Endoscopic } \\
\text { Diagnosis }\end{array}$ & $\begin{array}{c}\text { GERD } \\
\text { No (\%age) }\end{array}$ & $\begin{array}{c}\text { Erosive Gastritis } \\
\text { No (\%age) }\end{array}$ & $\begin{array}{c}\text { Gastric Ulcers } \\
\text { No (\%age) }\end{array}$ & $\begin{array}{c}\text { Duodenal Ulcers } \\
\text { No (\%age) }\end{array}$ \\
\hline Sequential & $20(40 \%)$ & $30(60 \%)$ & $16(32 \%)$ & $2(4 \%)$ \\
\hline Standard Triple & $17(34 \%)$ & $27(54 \%)$ & $20(40 \%)$ & $4(8 \%)$ \\
\hline Quinolone & $18(36 \%)$ & $42(84 \%)$ & $13(26 \%)$ & $1(2 \%)$ \\
\hline Total & $55(36.7 \%)$ & $99(66 \%)$ & $49(32.7 \%)$ & $7(4.7 \%)$ \\
\hline P value & $0.818 \mathrm{NS}$ & $0.004 *$ & $0.326 \mathrm{NS}$ & $0.350 \mathrm{NS}$ \\
\hline
\end{tabular}

NS; $p>0.05$; Not Significant; ${ }^{*} p<0.05$; Significant

Table 1 shows percentage distribution of endoscopic diagnosis in three groups. There was no significant difference with regards to presence of GERD, Gastric Ulcers, Duodenal Ulcers ( $p$ value> 0.05) except for presence of erosive gastritis which was significantly higher in patients in quinolone group ( $\mathrm{p}$ value $<0.05$ ).

Table 2 Comparison of follow-up rapid urease test of three groups

\begin{tabular}{|l|c|c|c|c|}
\hline \multirow{2}{*}{$\begin{array}{c}\text { Follow-up Rapid } \\
\text { Urease Test }\end{array}$} & $\begin{array}{c}\text { Sequential Therapy } \\
\text { No }(\% \text { age })\end{array}$ & $\begin{array}{c}\text { Triple Therapy } \\
\text { No }(\% \text { age })\end{array}$ & $\begin{array}{c}\text { Quinolone } \\
\text { Therapy } \\
\text { No (\%age) }\end{array}$ & $\begin{array}{c}\text { Total } \\
\text { No (\%age) }\end{array}$ \\
\hline Negative & $45(90 \%)$ & $43(86 \%)$ & $41(82 \%)$ & $129(86 \%)$ \\
\hline Positive & $5(10 \%)$ & $7(14 \%)$ & $9(18 \%)$ & $21(14 \%)$ \\
\hline Total & $50(100 \%)$ & $50(100 \%)$ & $50(100 \%)$ & $50(100 \%)$ \\
\hline \multicolumn{2}{|c|}{$\mathrm{x}^{2}=1.329 ; \mathrm{df}=2 ; \mathrm{p}=0.515(>0.05) ;$ Not Significant } & \\
\hline
\end{tabular}

Table 2 show that eradication rate was $90 \%$, $86 \%, 82 \%$ in sequential therapy group, triple therapy group and fluroquinolone group respectively. However, there was no statistically significant difference in eradication rates in these groups ('p'value>0.05).

Table 3 Side Effects of Patients in Three Groups after Treatment

\begin{tabular}{|l|c|c|c|c|c|c|}
\hline \multicolumn{1}{|c|}{ Groups } & $\begin{array}{c}\text { Taste } \\
(\%)\end{array}$ & $\begin{array}{c}\text { Abdominal } \\
\text { Pain }(\%)\end{array}$ & $\begin{array}{c}\text { Bloating } \\
(\%)\end{array}$ & $\begin{array}{c}\text { Nausea/ } \\
\text { Vomit }(\%)\end{array}$ & $\begin{array}{c}\text { Diarrhoea } \\
(\%)\end{array}$ & $\begin{array}{c}\text { Constipation } \\
(\%)\end{array}$ \\
\hline Sequential & $2(4 \%)$ & $1(2 \%)$ & $3(6 \%)$ & $4(8 \%)$ & $7(14 \%)$ & $1(2 \%)$ \\
\hline Standard Triple & $1(2 \%)$ & $2(4 \%)$ & $1(2 \%)$ & $6(12 \%)$ & $6(12 \%)$ & - \\
\hline Fluroquinolone & - & $2(4 \%)$ & $2(4 \%)$ & $4(8 \%)$ & $6(12 \%)$ & $1(2 \%)$ \\
\hline Total & $3(2 \%)$ & $5(3.3 \%)$ & $6(4 \%)$ & $14(9.3 \%)$ & $\begin{array}{c}19 \\
(12.7 \%)\end{array}$ & $2(1.3 \%)$ \\
\hline 'P' Value & $0.360 \mathrm{NS}$ & $0.813 \mathrm{NS}$ & $0.594 \mathrm{NS}$ & $0.730 \mathrm{NS}$ & $0.942 \mathrm{NS}$ & $0.602 \mathrm{NS}$ \\
\hline
\end{tabular}

NS; $p>0.05$; Not Significant 
Table 3 shows side effect profile in three groups at follow up. Diarrhoea was the most common side effect reported 12.7 percent and constipation was least common side effect 1.3 percent. There was no significant difference in side effect profile in three groups ' $p$ ' Value $>0.05$ for all the reported side effects.

Table 4 Comparison of follow-up rapid urease test in sequential vs standard triple therapy

\begin{tabular}{|l|c|c|c|}
\hline \multirow{2}{*}{$\begin{array}{c}\text { Follow-up Rapid } \\
\text { Urease Test }\end{array}$} & \multicolumn{2}{|c|}{ Group } & \multirow{2}{*}{$\begin{array}{c}\text { Total } \\
\text { No }(\%)\end{array}$} \\
\cline { 2 - 3 } & $\begin{array}{c}\text { Sequential Therapy } \\
\text { No (\%) }\end{array}$ & $\begin{array}{c}\text { Standard Triple } \\
\text { Therapy No }(\%)\end{array}$ & Negative \\
\hline Positive & $55(90 \%)$ & $43(86.0 \%)$ & $88(88 \%)$ \\
\hline Total & $5(10.0 \%)$ & $7(14.0 \%)$ & $12(12 \%)$ \\
\hline \multicolumn{2}{|r|}{$\mathrm{x}^{2}=0.379 ; \mathrm{df}=1 ; \mathrm{p}=0.538(>0.05) ;$ Not Significant } \\
\hline
\end{tabular}

Table 4 shows comparison of follow-up rapid urease test in sequential vs standard triple therapy group. Eradication rate in sequential group was 90 percent whereas in standard triple therapy group was 86 percent. However, this difference was not statistically significant ('p'value $>0.05$ ).

Table 5 Side effects of patients in sequential vs standard triple therapy groups

\begin{tabular}{|l|c|c|c|c|c|c|}
\hline \multicolumn{1}{|c|}{ Groups } & $\begin{array}{c}\text { Taste No } \\
(\%)\end{array}$ & $\begin{array}{c}\text { Abdominal } \\
\text { Pain No }(\%)\end{array}$ & $\begin{array}{c}\text { Bloating No } \\
(\%)\end{array}$ & $\begin{array}{c}\text { Nausea/ } \\
\text { Vomit no } \\
(\%)\end{array}$ & $\begin{array}{c}\text { Diarrhoea } \\
\text { No (\%) }\end{array}$ & $\begin{array}{c}\text { Constipation } \\
\text { No (\%) }\end{array}$ \\
\hline Sequential & $2(4 \%)$ & $1(2 \%)$ & $3(6 \%)$ & $4(8 \%)$ & $7(14 \%)$ & $1(2 \%)$ \\
\hline $\begin{array}{l}\text { Standard } \\
\text { Triple }\end{array}$ & $1(2 \%)$ & $2(4 \%)$ & $1(2 \%)$ & $6(12 \%)$ & $6(12 \%)$ & - \\
\hline Total & $3(3 \%)$ & $3(3 \%)$ & $4(4 \%)$ & $10(10 \%)$ & $13(13 \%)$ & $1(\%)$ \\
\hline 'P' Value & $0.558 \mathrm{NS}$ & $0.558 \mathrm{NS}$ & $0.307 \mathrm{NS}$ & $0.505 \mathrm{NS}$ & $0.766 \mathrm{NS}$ & $0.315 \mathrm{NS}$ \\
\hline
\end{tabular}

NS; $p>0.05$; Not Significant

Table 5 shows side effect profile in sequential vs standard triple therapy group. There was no significant difference in two groups in terms of side effects.

Table 6 Follow up rapid urease test comparison of sequential vs quinolone therapy

\begin{tabular}{|l|c|c|c|}
\hline \multirow{2}{*}{$\begin{array}{c}\text { Follow-up Rapid } \\
\text { Urease Test }\end{array}$} & \multicolumn{2}{|c|}{ Groups } & \multirow{2}{*}{\begin{tabular}{c} 
Total No (\%) \\
\cline { 2 - 3 }
\end{tabular}} \\
\cline { 2 - 3 } Negative & $45(90.0 \%)$ & $41(82 \%)$ & $86(86.0 \%)$ \\
\hline Positive & $5(10.0 \%)$ & $9(18.0 \%)$ & $14(14.0 \%)$ \\
\hline Total & $50(100.0 \%)$ & $50(100.0 \%)$ & $100(100.0 \%)$ \\
\hline
\end{tabular}

$\mathrm{x}^{2}=1.39 ; \mathrm{df}=1 ; \mathrm{p}=0.249(>0.05) ;$ Not Significant

Table 6 shows comparison of follow-up rapid urease test in sequential vs quinolone therapy group. Eradication rate in sequential group was 90 percent whereas in quinolone therapy group was 82 percent. However, this difference was not statistically significant ('p'value $>0.05$ ). 
Int. J. Curr. Res. Med. Sci. (2017). 3(10): 98-106

Table 7 Side effects of patients in sequential vs quinolone groups

\begin{tabular}{|l|c|c|c|c|c|c|}
\hline \multicolumn{1}{|c|}{ Groups } & $\begin{array}{c}\text { Taste } \\
\text { No }(\%)\end{array}$ & $\begin{array}{c}\text { Abdominal } \\
\text { Pain No }(\%)\end{array}$ & $\begin{array}{c}\text { Bloating } \\
\text { No }(\%)\end{array}$ & $\begin{array}{c}\text { Nausea/ } \\
\text { Vomit No } \\
(\%)\end{array}$ & $\begin{array}{c}\text { Diarrhoea } \\
\text { No (\%) }\end{array}$ & $\begin{array}{c}\text { Constip-ation } \\
\text { No (\%) }\end{array}$ \\
\hline Sequential & $2(4 \%)$ & $1(2 \%)$ & $3(6 \%)$ & $4(8 \%)$ & $7(14 \%)$ & $1(2 \%)$ \\
\hline Quinolone & - & $2(4 \%)$ & $2(4 \%)$ & $4(8 \%)$ & $6(12 \%)$ & $1(2 \%)$ \\
\hline Total & $2(2 \%)$ & $3(3 \%)$ & $5(5 \%)$ & $8(8 \%)$ & $13(13 \%)$ & $2(2 \%)$ \\
\hline 'P' Value & $0.153^{\mathrm{NS}}$ & $0.558^{\mathrm{NS}}$ & $0.646^{\mathrm{NS}}$ & $1^{\mathrm{NS}}$ & $0.766^{\mathrm{NS}}$ & $1^{\mathrm{NS}}$ \\
\hline
\end{tabular}

NS; $p>0.05$; Not Significant

Table 7 shows side effect profile in sequential vs

Quinolone therapy group. There was no

significant difference in two groups in terms of side effects ( $\mathrm{p}$ value $>0.05$ ).

Table 8 Follow-up rapid urease test comparison of triple therapy vs quinolone groups

\begin{tabular}{|l|c|c|c|}
\hline \multirow{2}{*}{$\begin{array}{c}\text { Follow-up Rapid Urease } \\
\text { Test }\end{array}$} & \multicolumn{2}{|c|}{ Groups } & \multirow{2}{*}{$\begin{array}{c}\text { Total } \\
\text { No }(\%)\end{array}$} \\
\cline { 2 - 3 } & Triple Therapy no (\%) & $\begin{array}{c}\text { Quinilone Therapy no } \\
(\%)\end{array}$ & $84.0 \%(\mathrm{n}=84)$ \\
\hline Negative & $43(86.0 \%)$ & $41(82 \%)$ & $16.0 \%(\mathrm{n}=16)$ \\
\hline Positive & $7(14.0 \%)$ & $9(18.0 \%)$ & $100.0(100 \%)$ \\
\hline Total & $50(100.0 \%)$ & $50(100.0 \%)$ & \\
\hline \multicolumn{2}{|c|}{$\mathrm{x}^{2}=0.298 ; \mathrm{df}=1 ; \mathrm{p}=0.585$} & $(>0.05) ;$ Not Significant \\
\hline
\end{tabular}

Table 8 shows comparison of follow-up rapid urease test in standard triple therapy group vs quinolone therapy group. Eradication rate in standard therapy group was 86 percent whereas in quinolone therapy group was 82 percent. However, this difference was not statistically significant ('p'value >0.05).

Table 9 Side effects of patients in standard triple therapy vs quinolone group

\begin{tabular}{|l|c|c|c|c|c|c|}
\hline \multicolumn{1}{|c|}{ Groups } & $\begin{array}{c}\text { Taste No } \\
(\%)\end{array}$ & $\begin{array}{c}\text { Abdominal } \\
\text { Pain No. (\%) }\end{array}$ & $\begin{array}{c}\text { Bloating No } \\
(\%)\end{array}$ & $\begin{array}{c}\text { Nausea/ } \\
\text { Vomit No } \\
(\%)\end{array}$ & $\begin{array}{c}\text { Diarrhoea } \\
\text { No (\%) }\end{array}$ & $\begin{array}{c}\text { Constip- } \\
\text { ation } \\
\text { No (\%) }\end{array}$ \\
\hline $\begin{array}{l}\text { Standard } \\
\text { Triple }\end{array}$ & $1(2 \%)$ & $2(4 \%)$ & $1(2 \%)$ & $6(12 \%)$ & $6(12 \%)$ & - \\
\hline Quinolone & - & $2(4 \%)$ & $2(4 \%)$ & $4(8 \%)$ & $6(12 \%)$ & $1(2 \%)$ \\
\hline Total & $1(1 \%)$ & $4(4 \%)$ & $3(3 \%)$ & $10(10 \%)$ & $12(12 \%)$ & $1(1 \%)$ \\
\hline 'P' Value & $0.315^{\mathrm{NS}}$ & $1^{\mathrm{NS}}$ & $0.558^{\mathrm{NS}}$ & $0.505^{\mathrm{NS}}$ & $1^{\mathrm{NS}}$ & $0.315^{\mathrm{NS}}$ \\
\hline
\end{tabular}

NS; $p>0.05$; Not Significant

Table 9 shows side effect profile in standard triple vs Quinolone therapy group. There was no significant difference in two groups in terms of side effects. 


\section{Discussion}

In our study, there was no significant difference in age distribution in all three groups. Mean age for sequential therapy group was 45.46 years, mean age for standard triple therapy group was 44.84 years and mean age for Quinolone Therapy group was 43.30. Mean age for all three groups was 44.53. There was no significant difference in sex distribution in three groups.

In our study eradication rate for sequential therapy group was 90 percent. This finding was consistent with study conducted by Vaira et $\mathrm{al}^{20}$ in Italy between 2003 and 2006 where eradication rate of 89 percent was obtained with sequential therapy. Similar results were obtained in a study conducted by Zullo et al. ${ }^{21}$ Eradication rate for 14 days standard triple therapy was 86 percent and this finding was consistent with results of study conducted by Yuan et $\mathrm{al}^{22}$ where eradication rate of 84.4 percent was obtained with 14 days standard triple therapy. Eradication rate for 10 days fluroquinolone based triple therapy was 82 percent. This finding was consistent with results of study conducted by Gisbert et $\mathrm{al}^{23}$ where eradication rate of 83 percent was obtained with levofloxacin containing regimen.

Eradication rate was slightly higher in sequential therapy group (90 percent) compared to standard triple therapy group (86 percent) or fluroquinolone based triple therapy group (82 percent), however the results were statistically insignificant ( $p$ value $>0.05$ ) when all three groups were compared together suggesting all three regimens were equivalent in terms of achieving Helicobacter Pylori eradication. No significant difference was found in terms of side effect profile in three groups. There was no significant difference in eradication rates or side effect profile when sequential therapy group was compared with triple therapy group. There was no significant difference in eradication rates or side effect profile when sequential therapy group was compared with fluroquinolone based triple therapy group. There was no significant difference in eradication rates or side effect profile when standard triple therapy group was compared with fluroquinolone based triple therapy group. In all three groups diarrhoea was most common side effect reported (mean 12.7\%). It was followed by nausea/vomit (mean 9.3\%).

These findings were consistent with a multicenter randomised control trial where in 10 days sequential therapy was compared with 14 days Triple Therapy conducted by Liou JM. ${ }^{24}$ No difference was noted in eradication rates or adverse effects in two groups. Also, a metaanalysis and systematic review consisting of 46 randomised controlled trials concluded that eradication rates in 10 days sequential therapy and 14 days standard triple therapy were statistically insignificant. $^{25}$

Similarly results in our study were consistent with results of a study conducted by J. Molina-Infante where levofloxacin based therapy was compared with sequential therapy. No difference was found with respect to eradication in two groups. ${ }^{26}$

Our results were consistent with a recent meta analysis where seven trials were identified with 888 patients receiving first-line levofloxacin and 894 treated with standard therapy (Amoxicillin, Clarithromycin and proton pump inhibitor). This metaanalysis concluded that Helicobacter pylori eradication rates with Levofloxacin-based first line therapy had equivalent results as that of standard first-line therapy. ${ }^{21}$

\section{References}

1. Brown LM. Helicobacter pylori: epidemiology and routes of transmission. Epidemiol Rev.2000; 22 (2):283-97.

2. Eidt S, Stolte M, Fischer RJ. Helicobacter pylori gastritis and primary gastric nonHodgkin's lymphomas. Clin Pathol 1994; 47 (5):436-9.

3. Wündisch T, Thiede C, Morgner A, Dempfle A, Günther A, Liu H, Ye H, Du MQ, Kim TD, Bayerdörffer E, Stolte M, Neubauer A. Longterm follow-up of gastric MALT lymphoma after Helicobacter pylori eradication. J Clin Oncol. 2005; 23 (31):8018-24. 
4. Bauerfeind P, Garner R, Dunn BE, Mobley HL. Synthesis and activity of Helicobacter pylori urease and catalase at low Ph. Gut. 1997; 40 (1):25-30.

5. Perez GI, Rothenbacher D, and H. Brenner. Epidemiology of Helicobacter pylori infection. Helicobacter.2004; 9 (1):1-6.

6. Konno M, Fujii N, Yokota S, Sato K, Takahashi M, Mino E, Sugiyama T: Five-year follow-up study of mother-to-child transmission of Helicobacter pylori infection detected by a random amplified polymorphic DNA fingerprinting method. J Clin Microbiol 2005; 43:2246-50.

7. Johannes G. Kusters, Arnoud H. M. van Vliet, Ernst J. Kuipers. Pathogenesis of Helicobacter pylori Infection. Clin Microbiol Rev. 2006;19 (3): 449-90.

8. Ciociola AA, McSorley DJ, Turner K. Helicobacter pylori infection rates in duodenal ulcer patients in the United States may be lower than previously estimated. Am J Gastroenterol 1999; 94:1834-40.

9. Malfertheiner P, Megraud F, O'Morain CA, Gisbert JP, Kuipers EJ, Axon AT et. al. Management of Helicobacter pylori infectionthe Maastricht V/Florence Consensus Report. Gut. 2017; 66 (1):6-30. [Medline].

10. Chey WD, Leontiadis GI, Howden CW, Moss SF. ACG Clinical Guideline: Treatment of Helicobacter pylori Infection. Am J

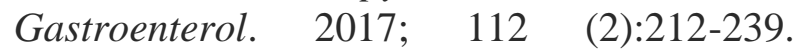
[Medline]

11. Nishizawa $T$, Maekawa $T$, Watanabe $N$, Harada N, Hosoda $\mathrm{Y}$, Yoshinaga $\mathrm{M}$, et al. Clarithromycin Versus Metronidazole as Firstline Helicobacter pylori Eradication: A Multicenter, Prospective, Randomized Controlled Study in Japan. J Clin Gastroenterol. 2015; 49 (6):468-71. [Medline]. 12. Nyssen OP, McNicholl AG, Megraud F, Savarino V, Oderda G, Fallone CA, et al. Sequential versus standard triple first-line therapy for Helicobacter pylori eradication. Cochrane Database Syst Rev. 2016 Jun 28. CD009034. [Medline].

13.Liou JM, Fang YJ, Chen CC, Bair MJ, Chang CY, Lee YC et. al. Concomitant, bismuth quadruple, and 14-day triple therapy in the first-line treatment of Helicobacter pylori: a multicentre, open-label, randomised trial. Lancet. 2016 Nov 12. 388 (10058):23552365. [Medline].

14.Vakil N, Megraud F. Eradication therapy for Helicobacter pylori. Gastroenterology. 2007; 133:985-1001

15. Molina-Infante J, Lucendo AJ, Angueira T, Rodriguez-Tellez M, Perez-Aisa A, Balboa A, et al. Optimised empiric triple and concomitant therapy for Helicobacter pylori eradication in clinical practice: the OPTRICON study. Aliment Pharmacol Ther. 2015 ; 41 (6):581-89. [Medline].

16. Kale-Pradhan PB, Mihaescu A, Wilhelm SM. Fluoroquinolone Sequential Therapy for Helicobacter pylori: A Meta-analysis. Pharmacotherapy. 2015; 35 (8):719-30. [Medline].

17. Gatta L, Vakil N, Leandro G. Sequential therapy or triple therapy for Helicobacter pylori: Systematic review and meta-analysis of randomized controlled trials in adult and children. Am J Gastroenterol. 2009;104:306979.

18. Gisbert JP, Pajares JM. Treatment of Helicobacter pylori infection: the past and the future. Eur J Intern Med. 2010; 21:357-359.

19. Gisbert JP, Bermejo MF, Infante JM, Gallardo $\mathrm{BP}$, Bermejo AB, Rodríguez JM, Andrés PR, García GG. Levofloxacin, Amoxicillin, and Omeprazole as first-line triple therapy for Helicobacter pylori eradication. J Clin Gastroenterol. 2009; 43 (4):384-5.

20. Vaira D, Zullo A, Vakil N, Gatta L, Ricci C, Perna F, et al. Sequential Therapy versus Standard Triple-Drug Therapy for Helicobacter pylori Eradication A Randomized Trial. Ann Intern Med. 2007; 146(8):556-63.

21. Peedikayil MC, AlSohaibani FI, Alkhenizan AH. Levofloxacin-Based First-Line Therapy versus Standard of Family st Hospital \& Research Center, Riyadh, Kin First-Line Therapy for Helicobacter pylori Eradication: Meta-Analysis of Randomized Controlled Trials. PLoS ONE 2014; 9(1): e85620. 
22. Yuan Y, Ford AC, Khan KJ, Gisbert JP, Forman D, Leontiadis GI, et al. Optimum duration of regimens for Helicobacter pylori eradication. Cochrane Database Syst Rev. 2013; 12: CD008337.

23. Gisbert JP, Bermejo MF, Infante JM, Gallardo $\mathrm{BP}$, Bermejo $\mathrm{AB}$, Rodriguez $\mathrm{JM}$, et al. Levofloxacin, amoxicillin, and omeprazole as first line triple therapy or Helicobactor pylori eradication. J Clin Gastroentrol. 2009; 43: 3845 .

24. Liou JM, Chen CC, Chen MJ, et al. Sequential versus triple therapy for the firstline treatment of Helicobacter pylori: a multicentre, open-label, randomised trial. Lancet 2013; 381: 205-13

25. Gatta L, Scarpignato C, Vakil N, Vaira D. Global eradication rates for Helicobacter pylori infection: systematic review and metaanalysis of sequential therapy. Brit Med J. 2013; 347: f4587.

26. Molina-Infante J, Perez-Gallardo B, Fernandez-Bermejo M, Hernandez-Alonso M, Vinagre G, Dueñas $\mathrm{C}$, et al. Clinical Trial: Clarithromycin vs. Levofloxacin in First-line Triple and Sequential Regimens for Helicobacter pylori Eradication. Aliment Pharmacol Ther. 2010; 31(10): 1077-84.

\begin{tabular}{|c|c|}
\hline \multicolumn{2}{|c|}{ Access this Article in Online } \\
\hline 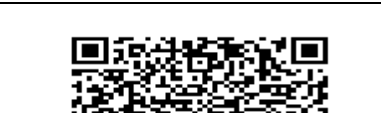 & $\begin{array}{l}\text { Website: } \\
\text { www.ijcrims.com }\end{array}$ \\
\hline 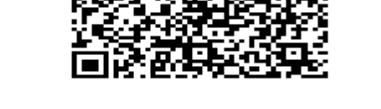 & \multirow[t]{2}{*}{$\begin{array}{l}\text { Subject: } \\
\text { Medicine }\end{array}$} \\
\hline Quick Response Code & \\
\hline
\end{tabular}

How to cite this article:

Krishan kumar Oberoi, Upasana. (2017). Comparative study of sequential therapy versus standard triple therapy versus quinolone-based triple therapy for eradication of Helicobacter pylori infection. Int. J. Curr. Res. Med. Sci. 3(10): 98-106.

DOI: http://dx.doi.org/10.22192/ijcrms.2017.03.10.014 